\title{
LINEAR DILATATION AND DIFFERENTIABILITY OF HOMEOMORPHISMS OF $\mathbb{R}^{n}$
}

\author{
BRUCE HANSON \\ (Communicated by Mario Bonk) \\ In memory of Juha Heinonen
}

\begin{abstract}
According to a classical result, if $\Omega$ is a domain in $\mathbb{R}^{d}$, where $d>1, f: \Omega \rightarrow \mathbb{R}^{d}$ is a homeomorphism and the lim-sup dilatation $H_{f}$ of $f$ is finite almost everywhere on $\Omega$, then $f$ is differentiable almost everywhere on $\Omega$. We show that this theorem fails if $H_{f}$ is replaced by the lim-inf dilatation $h_{f}$. Our example demonstrates the sharpness of recent results of Kallunki and Koskela concerning the $h_{f}$ function and also of Balogh and Csörnyei involving the lower-scaled oscillation of continuous functions $f: \Omega \rightarrow \mathbb{R}$.
\end{abstract}

\section{Statement of MAin Result}

Throughout this section we assume that $\Omega$ is a domain in $\mathbb{R}^{d}$, where $d \geq 2$ and $f: \Omega \rightarrow \Omega^{\prime} \subset \mathbb{R}^{d}$ is a homeomorphism. For any $x$ in $\Omega$ the linear lim sup and lim inf dilatations of $f$ at $x$ are defined by

$$
\begin{gathered}
H_{f}(x)=\limsup _{r \rightarrow 0} H_{f}(x, r)=\limsup _{r \rightarrow 0} \frac{L_{f}(x, r)}{l_{f}(x, r)}, \\
h_{f}(x)=\liminf _{r \rightarrow 0} H_{f}(x, r)=\liminf _{r \rightarrow 0} \frac{L_{f}(x, r)}{l_{f}(x, r)},
\end{gathered}
$$

where

$$
\begin{gathered}
L_{f}(x, r)=\max \{|f(x)-f(y)|:|x-y| \leq r\}, \\
l_{f}(x, r)=\min \{|f(x)-f(y)|:|x-y| \geq r\} .
\end{gathered}
$$

The $H_{f}$ function is intimately connected with the theory of quasiconformal mappings as illustrated by the following well-known classical result (see, for example, [V], Theorem 34.1):

Theorem 1.1. If there exists $K<\infty$ such that $H_{f}(x) \leq K$ for all $x \in \Omega$, then $f$ is quasiconformal on $\Omega$.

In [G] Gehring showed that the hypotheses on $H_{f}$ can be weakened a bit and still give the same conclusion as in Theorem 1.1

Theorem 1.2. Suppose that $S$ is a subset of $\Omega$ with $\sigma$-finite $(d-1)$-dimensional measure, $H_{f}(x)<\infty$ for all $x \in \Omega \backslash S$ and there is a $K<\infty$ such that $H_{f}(x) \leq K$ a.e. on $\Omega$. Then $f$ is quasiconformal on $\Omega$.

Received by the editors April 12, 2011.

2010 Mathematics Subject Classification. Primary 30C65; Secondary 26B05. 
Another way to weaken the hypotheses in Theorem 1.1 is by replacing $H_{f}$ with $h_{f}$. In [HK] Heinonen and Koskela showed that $H_{f}$ can be replaced with $h_{f}$ in Theorem 1.1.

Theorem 1.3. Suppose there exists $K<\infty$ such that

$$
h_{f}(x) \leq K
$$

for all $x \in \Omega$. Then $f$ is quasiconformal on $\Omega$.

More recently Koskela and Kallunki $[\mathrm{KK}$ showed that Theorem 1.2 is also true with $H_{f}$ replaced by $h_{f}$ :

Theorem 1.4. Suppose that $S$ is a subset of $\Omega$ with $\sigma$-finite $(d-1)$-dimensional measure, $h_{f}(x)<\infty$ for all $x \in \Omega \backslash S$ and there is a $K<\infty$ such that $h_{f}(x) \leq K$ a.e. on $\Omega$. Then $f$ is quasiconformal on $\Omega$.

Since quasiconformal mappings are almost everywhere differentiable, it follows that the hypotheses of Theorem 1.2 imply that $f$ is differentiable a.e. on $\Omega$. In fact, one can significantly weaken the hypotheses on $H_{f}$ and still get the a.e. differentiability of $f$ :

Theorem 1.5. Suppose that $H_{f}(x)<\infty$ a.e. on $\Omega$. Then $f$ is differentiable a.e. on $\Omega$.

Theorem 1.5 can be found, for example, in $[\mathrm{KM}$ and is an easy consequence of the following versions of two classical theorems:

Theorem 1.6 (Rademacher-Stepanov). Define

$$
L_{f}(x)=\limsup _{r \rightarrow 0} \frac{L_{f}(x, r)}{r} .
$$

If $L_{f}(x)<\infty$ a.e. on $\Omega$, then $f$ is a.e. differentiable on $\Omega$.

Theorem 1.7 (Lebesgue Differentiation). For almost every $x \in \Omega$ the following limit exists:

$$
\lim _{r \rightarrow 0} \frac{|f(B(x, r))|}{|B(x, r)|} .
$$

Note that for each of these last two results we are still assuming that $f$ is a homeomorphism from $\Omega$ into $\mathbb{R}^{d}$, a much stronger hypothesis than is required in either case. A natural question to ask at this point is whether we can replace $H_{f}$ with $h_{f}$ in Theorem 1.5. The answer to this question is an emphatic no, as our following main result shows.

Theorem 1.8. Suppose that $d \geq 2$. Let $h:[0, \infty) \rightarrow[0, \infty)$ be a homeomorphism satisfying

$$
\frac{h(t)}{t^{d-1}} \rightarrow 0 \text { as } t \rightarrow 0^{+} .
$$

Then there exists a homeomorphism $f:[0,1]^{d} \rightarrow f\left([0,1]^{d}\right) \subset \mathbb{R}^{d}$ and a set $S \subset$ $[0,1]^{d}$ such that

$$
\begin{gathered}
\mathcal{H}^{h}(S)=0, \\
h_{f}(x)=1 \text { for all } x \in[0,1]^{d} \backslash S,
\end{gathered}
$$




$$
H_{f}(x)=\infty \text { for all } x \in[0,1]^{d},
$$

$$
f \text { is nowhere differentiable on }[0,1]^{d} \text {. }
$$

The notation denotes the Hausdorff measure with respect to $h$ and will be defined precisely in section three. Note that if $h(t)=\frac{t^{d-1}}{-\log t}$ for small $t$, then $h$ satisfies (7) and in this case $S$ has Hausdorff dimension no larger than $d-1$. This demonstrates that Theorem 1.4 is quite sharp even regarding differentiability.

\section{SCALED Oscillation}

In this section we again assume that $\Omega$ is a domain in $\mathbb{R}^{d}$, but we only require that $d \geq 1$ and we assume that $f: \Omega \rightarrow \mathbb{R}$ is continuous. We note that Theorem 1.6 remains true in this setting. In $[\mathrm{BC}]$ Balogh and Csörnyei consider analogues of this theorem and show that it is false if one replaces the upper scaled oscillation function $L_{f}$ (defined in (6) ) with the lower scaled oscillation function $l_{f}$, defined by:

$$
l_{f}(x)=\liminf _{r \rightarrow 0} \frac{L_{f}(x, r)}{r} .
$$

In fact, they show that

Theorem 2.1. For every $d \geq 1$ there exists a nowhere differentiable, continuous function $f:[0,1]^{d} \rightarrow \mathbb{R}$ such that $l_{f}(x)=0$ for a.e. $x \in[0,1]^{d}$.

Note that to obtain Theorem 2.1 it suffices to prove that there exists a nowhere differentiable, continuous function $g:[0,1] \rightarrow \mathbb{R}$ such that $l_{g}(x)=0$ for almost every $x \in[0,1]$. The higher-dimensional case then follows by considering the function $f:[0,1]^{d} \rightarrow \mathbb{R}$ defined by $f\left(x_{1}, x_{2}, \ldots, x_{d}\right)=g\left(x_{1}\right)$. In the positive direction, by strengthening the hypotheses on $l_{f}$, Balogh and Csörnyei obtain the following:

Theorem 2.2. Assume that $l_{f}(x)<\infty$ on $\Omega \backslash E$ where $E$ has $\sigma$-finite $(d-1)$ dimensional Hausdorff measure. Assume also that $l_{f} \in L_{l o c}^{p}(\Omega)$ where $p>d$ if $d>1$ and $p=1$ if $d=1$. Then $f$ is differentiable a.e. on $\Omega$.

Theorem 2.1 shows that one cannot weaken the hypotheses in Theorem 2.2 by requiring only that the exceptional set $E$ have $d$-dimensional Lebesgue measure 0 . Our next result strengthens Theorem 2.1 showing that, in fact, $E$ can have Hausdorff dimension at most $d-1$ in Theorem 2.2.

Theorem 2.3. Suppose that $d \geq 1$. Let $h:[0, \infty) \rightarrow[0, \infty)$ be a homeomorphism satisfying (7). Then there exist a continuous function $f:[0,1]^{d} \rightarrow \mathbb{R}$ and a set $S \subset[0,1]^{d}$ such that (15) and (11) hold and

$$
\begin{gathered}
l_{f}(x)=0 \text { for all } x \in[0,1]^{d} \backslash S, \\
L_{f}(x)=\infty \text { for all } x \in[0,1]^{d} .
\end{gathered}
$$




\section{HAUSDORFF MEASURE}

Let $h:[0, \infty) \rightarrow[0, \infty)$ be a homeomorphism. For each $\delta>0$ and $A \subset \mathbb{R}^{d}$ define

$$
\mathcal{H}_{\delta}^{h}(A)=\inf \sum_{i} h\left(\operatorname{diam} E_{i}\right)
$$

where the infimum is taken over all sequences $\left(E_{i}\right)$ of subsets of $\mathbb{R}^{d}$ such that $A \subset \bigcup_{i} E_{i}$ and each $E_{i}$ has diameter $\leq \delta$. Note that $\mathcal{H}_{\delta}^{h}$ is a decreasing function of $\delta$, so we can define

$$
\mathcal{H}^{h}(A)=\lim _{\delta \rightarrow 0} \mathcal{H}_{\delta}^{h}(A)=\sup _{\delta>0} \mathcal{H}_{\delta}^{h}(A) .
$$

Note that in the case that $h(t)=t^{d}$, where $d>0$, then $\mathcal{H}^{h}(A)$ is just the standard $d$-dimensional Hausdorff measure of $A$.

We are now ready to prove Theorems 1.8 and 2.3

\section{Proofs of Theorems 1.8 and 2.3}

We first note that the proofs of Theorems 1.8 and 2.3 follow rather quickly once we have established that Theorem 2.3 holds in the case $d=1$. To see this assume for the moment that we have proved Theorem 2.3 for this case. Fix $d>1$ and assume that $h$ is a homeomorphism of $[0, \infty)$ onto itself satisfying (7). For $0<t \leq 1$ define

$$
h^{*}(t)=\sup _{0<s \leq t} \frac{h(s)}{s^{d-1}}
$$

and for $t>1$ define $h^{*}(t)=t h^{*}(1)$. It follows that $h^{*}$ is an increasing homeomorphism of $(0, \infty)$ onto itself, and thus by our assumption we can find a continuous function $g:[0,1] \rightarrow \mathbb{R}$ and a set $E \subset[0,1]$ such that

$$
\begin{gathered}
\mathcal{H}^{h^{*}}(E)=0, \\
l_{g}(x)=0 \text { for all } x \in[0,1] \backslash E, \\
L_{g}(x)=\infty \text { for all } x \in[0,1],
\end{gathered}
$$

$g$ is nowhere differentiable on $[0,1]$.

To prove Theorem 1.8 define $f\left(x_{1}, x_{2}, \ldots, x_{d}\right)=\left(x_{1}, x_{2}, \ldots, x_{d-1}, x_{d}+g\left(x_{1}\right)\right)$ and $S=E \times[0,1]^{d-1}$. The continuity of $g$ guarantees that $f$ is a homeomorphism. Moreover, it is straighforward to show that (8) follows from (15) and also that (9), (10) and (11) follow from (16), (17) and (18).

For the proof of Theorem 2.3 we define $S$ as in the preceding paragraph, but now define $f\left(x_{1}, x_{2}, \ldots, x_{d}\right)=g\left(x_{1}\right)$. Then (11) again follows from the nowhere differentiability of $g$ and (13) and (14) follow easily from (16) and (17).

It remains to show that we can find a function $g$ and a set $E \subset[0,1]$ such that (15) -(18) all hold. The construction of $g$ is a variation of a standard construction of a nowhere differentiable, continuous function. To begin we need a bit of notation and a simple lemma.

For any nonnegative integer $n$ define $\mathcal{I}_{n}=\left\{I_{s, n}: s=0,1, \ldots, 2^{n}-1\right\}$, where $I_{s, n}=\left[s 2^{-n},(s+1) 2^{-n}\right]$. Given nonnegative integers $j$ and $k$, we say that $f$ is $(j, k)$-dyadic if $f$ is continuous on $[0,1]$ and for each $I \in \mathcal{I}_{j}, f$ is linear on $I$ with $f(I) \in \mathcal{I}_{k}$. 
Definition 4.1. Let $j$ and $k$ be nonnegative integers and $f$ be $(j, k)$-dyadic. Suppose that $l$ and $m$ are integers satisfying

$$
m \geq k+2 \text { and } l-m>j-k \text {. }
$$

Then the $(l, m)$-successor of $f$ is the unique $(l, m)$-dyadic function $g$ that satisfies the following three conditions:

$$
g\left(s 2^{-j}\right)=f\left(s 2^{-j}\right) \text { for } s=0,1,2, \ldots, 2^{j} .
$$

For each $I=I_{s, j} \in \mathcal{I}_{j}, g$ is linear on the intervals $\left[s 2^{-j}, s 2^{-j}+2^{m-l-k-1}\right]$ and $\left[(s+1) 2^{-j}-2^{m-l-k-1},(s+1) 2^{-j}\right]$ with slope the same sign as the sign of the slope of $f$ on $I$.

For each $I=I_{s, j} \in \mathcal{I}_{j}$, the sign of the slope of $g$ is $(-1)^{t}$ on each interval $I_{t, l} \subset I^{\prime}=\left[s 2^{-j}+2^{m-l-k-1},(s+1) 2^{-j}-2^{m-l-k-1}\right]$.

We then have the following lemma, which requires no proof:

Lemma 4.2. Suppose that $j, k, l, m$ satisfy (19), $f i s(j, k)$-dyadic and $g$ is the $(l, m)$-successor of $f$.

Then

$$
\begin{gathered}
|f(x)-g(x)| \leq 2^{-k} \text { for all } x \in[0,1], \\
\forall I \in \mathcal{I}_{j}|g(x)-g(y)| \leq 2^{-m} \text { for all } x, y \in I^{\prime} .
\end{gathered}
$$

We now begin the construction of $g$. We first choose $\left(j_{n}\right)$ to be an increasing sequence of nonnegative integers divisible by 3 and satisfying the following:

$$
\begin{gathered}
j_{0}=0, \\
j_{n+1} \geq 3 j_{n}+3 \forall n=0,1,2, \ldots, \\
h^{*}\left(2^{\frac{-j_{n+1}}{3}}\right) \leq 2^{-j_{n}-n-1} .
\end{gathered}
$$

For each $n=0,1,2, \ldots$ define $k_{n}=\frac{2 j_{n}}{3}$.

Let $g_{0}(x)=x$. Note that for all $n=0,1,2, \ldots$ we have $k_{n+1} \geq k_{n}+2$ and $j_{n+1}-k_{n+1}>j_{n}-k_{n}$. Thus, we can use Lemma 4.2 to inductively define a sequence of functions $\left(g_{n}\right)$ defined on $[0,1]$ such that for each $n=0,1,2, \ldots, g_{n+1}$ is the $\left(j_{n+1}, k_{n+1}\right)$-successor of $g_{n}$. Since $\left(k_{n}\right)$ is strictly increasing and $\lim _{n \rightarrow \infty} k_{n}=\infty$, it follows from (21) that $\left(g_{n}\right)$ converges uniformly on $[0,1]$ to a continuous function $g$.

We next show that (17) holds. Let $x \in[0,1]$. Given a positive integer $i$, choose $s_{i}$ such that $x \in I_{s_{i}, j_{i}}=\left[a_{i}, b_{i}\right]$. By construction of $g$ we have

It follows that

$$
\left|\frac{g\left(b_{i}\right)-g\left(a_{i}\right)}{b_{i}-a_{i}}\right|=\frac{2^{-k_{i}}}{2^{-j_{i}}}=2^{\frac{1}{2} k_{i}} .
$$

$$
\max \left\{\left|\frac{g\left(b_{i}\right)-g(x)}{b_{i}-x}\right|,\left|\frac{g(x)-g\left(a_{i}\right)}{x-a_{i}}\right|\right\} \geq 2^{\frac{1}{2} k_{i}} .
$$

Since $2^{\frac{1}{2} k_{i}} \rightarrow \infty$ and $b_{i}-a_{i} \rightarrow 0$, we see that $L_{g}(x)=\infty$. Thus, (17) is established and (18) clearly follows from (17).

We now move on to the proof of (15) and (16). For each $s=0,1,2, \ldots, 2^{j_{n}}-1$ define

$$
I_{s, j_{n}}^{\prime \prime}=\left[s 2^{-j_{n}}+2^{-\frac{1}{2} k_{n+1}-k_{n}},(s+1) 2^{-j_{n}}-2^{-\frac{1}{2} k_{n+1}-k_{n}}\right] .
$$


Note that if $x \in I_{s, j_{n}}^{\prime \prime}$ and $|x-y| \leq 2^{-\frac{1}{2} k_{n+1}-k_{n}-1}$, then $x, y \in I_{s, j_{n}}^{\prime}=\left[s 2^{-j_{n}}+\right.$ $\left.2^{-\frac{1}{2} k_{n+1}-k_{n}-1},(s+1) 2^{-j_{n}}-2^{-\frac{1}{2} k_{n+1}-k_{n}-1}\right]$, and hence by (22) we have

$$
\left|g_{n+1}(x)-g_{n+1}(y)\right| \leq 2^{-k_{n+1}} .
$$

Moreover, from the construction of $g$ it is easy to see that we can replace $g_{n+1}$ with $g$ in the inequality above. Summing up, we have:

$$
\text { If } x \in I_{s, j_{n}}^{\prime \prime} \text { and }|x-y| \leq 2^{-\frac{1}{2} k_{n+1}-k_{n}-1} \text {, then }|g(x)-g(y)| \leq 2^{-k_{n+1}} \text {. }
$$

Now for each positive integer $n$, define

$$
\begin{aligned}
& F_{n}=\bigcup_{s=0}^{2^{j} n} I_{s, j_{n}}^{\prime \prime}, \\
& F=\bigcap_{n=1}^{\infty}\left(\bigcup_{k=n}^{\infty} F_{k}\right)
\end{aligned}
$$

and let $E=[0,1] \backslash F$. Fix $x_{0} \in F=[0,1] \backslash E$. Then there is an increasing sequence of integers $\left(n_{i}\right)$ such that $x_{0} \in F_{n_{i}}$ for $i=1,2, \ldots$. Fix $i$ and choose $s$ such that $x_{0} \in I_{s, n_{i}}^{\prime \prime}$. Let $r=2^{-\frac{1}{2} k_{n_{i}+1}-k_{n_{i}}-1}$ and assume that $\left|x_{0}-x\right| \leq r$. Using (26), we get $\left|g\left(x_{0}\right)-g(x)\right| \leq 2^{-k_{n_{i}+1}}$, and a simple calculation using (24) shows that

$$
\frac{\left|g\left(x_{0}\right)-g(x)\right|}{r} \leq 2^{-\frac{j_{n_{i}}}{3}} .
$$

Since $2^{-\frac{j_{n_{i}}}{3}} \rightarrow 0$, we have (16) , as desired.

To complete the proof we need to establish (15). To that end define $E_{n}=$ $[0,1] \backslash F_{n}$ so that

$$
E=\bigcup_{n=1}^{\infty}\left(\bigcap_{k=n}^{\infty} E_{k}\right)
$$

where

$$
E_{n}=\bigcup_{s=0}^{2^{j_{n}}-1}\left(I_{s, j_{n}} \backslash I_{s, j_{n}}^{\prime \prime}\right) .
$$

Note that each $E_{n}$ is the union of $2^{j_{n}+1}$ intervals of length $2^{-\frac{1}{2} k_{n+1}-k_{n}}$. Since $k_{n} \geq 1$ for $n \geq 1$, the diameter of each of these intervals is less than $2^{\frac{-j_{n+1}}{3}}$, and thus by (25) we have

$$
\mathcal{H}_{\delta_{n}}^{h^{*}}\left(E_{n}\right) \leq 2^{j_{n}+1} h^{*}\left(2^{-\frac{j_{n+1}}{3}}\right) \leq 2^{-n}
$$

where $\delta_{n}=2^{\frac{-j_{n+1}}{3}}$. This confirms that (15) holds, and we are done with the proof.

\section{ACKNOWLEDGEMENT}

The author would like to thank Thomas Zürcher for carefully reading a preliminary version of the paper and for making many useful suggestions for improving it. 


\section{REFERENCES}

[BC] Balogh, Z., and M. Csörnyei, Scaled oscillation and regularity, Proc. Amer. Math. Soc. 134 9 (2006), 2667-2675. MR2213746 (2007b:46052)

[G] Gehring, F.W., Rings and quasiconformal mappings in space, Trans. Amer. Math. Soc. 103 (1962), 353-393. MR0139735(25:3166)

[HK] Heinonen, J., and P. Koskela, Definitions of quasiconformality, Invent. Math. 120 (1995), 61-79. MR.1323982 (96e:30051)

[KK] Kallunki, S., and P. Koskela, Exceptional sets for the definition of quasiconformality, American Journal of Mathematics 122 (2000), 735-743. MR1771571 (2001h:37095)

$[\mathrm{KM}]$ Kallunki, S., and O. Martio, ACL homeomorphisms and linear dilatation, Proc. Amer. Math. Soc. 130 (2002), 1073-1078. MR1873781 (2002i:30022)

[V] Väisälä, J., Lectures on n-Dimensional Quasiconformal Mappings. Lecture Notes in Mathematics, No. 229, Springer-Verlag, New York, 1971. MR.0454009 (56:12260)

Department of Mathematics, Statistics and Computer Science, St. Olaf College, Northfield, Minnesota 55057

E-mail address: hansonb@stolaf.edu 